\title{
L'APPROCCIO COMPARATISTICO NELLE DOTTRINE PROCESSUAL-PENALISTICHE. SPUNTI DI RIFLESSIONE IN ORDINE AL CONTESTO ITALIANO
}

\author{
Renzo ORLANDI \\ Università di Bologna \\ renzo.orlandi@unibo.it
}

SOMMARIO: 1. Premessa.-2. Realtà rilevanti.-3. Finalità della comparazione (in ambito processuale penale).-4. Gli oggetti della comparazione.-5. Utilità attuale della comparazione, nuovi compiti e conclusione.

1 Premessa. - L'approccio comparatistico ha un modesto seguito nella cultura processualpenalistica italiana. L'affermazione può suonare strana a chi coltiva la convinzione che la riforma processuale del 1988 si sia ispirata al sistema adversary e abbia avuto come modello l'esperienza nordamericana. In realtà, la storia di quella riforma è meno lineare di quel che a prima vista appare e sarebbe semplicistico rappresentarla come semplice importazione di un modello straniero ${ }^{1}$. Ciò che colpisce nella produzione scientifica del processualpenalista italiano è, anzi, il tendenziale disinteresse se non addirittura l'aperta diffidenza verso la cultura della comparazione. Uno stimato

\footnotetext{
1 Per più precise informazioni sul lungo e tortuoso percorso sfociato nella riforma del codice di procedura penale (1988) sia consentito rinviare a R. Orlandi, The Italian Path to Reform: Italy's Adversarial Model of Criminal Procedure, in Italian Law Journal 2019, p. 565 ss, Si veda altresì il precedente Diritti individuali e processo penale nell'Italia repubblicana, in D. Negri- M. Pifferi, Diritti individuali e processo penale nell'Italia repubblicana, Giuffrè, Milano, 2011. Anche per E. Amodio (Affermazioni e sconfitte della cultura dei giuristi nella elaborazione del nuovo codice di procedura penale, in Riv. it. dir e proc. pen. 1996, p. 899 ss.) la riforma processuale italiana del 1988 ha subito in misura marginale l'influsso della cultura di common law.
} 
autore mostra apertamente questa idiosincrasia: dopo aver osservato che "la nostra tradizione, la nostra struttura processuale, la nostra cultura, la nostra ideologia del processo è profondamente diversa da quella di altri Paesi" conclude che non vale la pena sforzarsi a "comparare elementi che non dovrebbero essere confusi" e aggiunge infine, con tono concessivo: "il che non vuol dire che non si possano adattare alla nostra tradizione istituti desunti da altre esperienze giudiziarie" ${ }^{2}$. Ovviamente, chi compara deve evitare parallelismi e ingenue sovrapposizioni: ma è proprio nell'intelligente uso di questa abilità di discernimento che sta il valore e, oserei dire, il vantaggio della comparazione rispetto allo studio circoscritto al diritto "nazionale". Peraltro, non si capisce sulla base di quale conoscenza (se non di origine comparatistica) si giustificherebbe quell'apertura agli “istituti di altre esperienze straniere”.

Le parole sopra riportate rivelano un sentimento diffuso fra gli studiosi italiani del processo penale: quanto meno con riguardo all'epoca che ci sta alle spalle. Si aggiunga che, in passato, la comparazione ha avuto scarso riconoscimento in ambito concorsuale. Presentare una monografia dove si ragionava su esperienze straniere poteva addirittura essere considerato un punto a sfavore, quasi si trattasse di una inutile esibizione. Non c'è quindi da sorprendersi se molti processual-penalisti hanno escluso dal loro orizzonte la curiosità per altri ordinamenti, per altre culture giuridiche, per altri modi di affrontare questioni cruciali, non dissimili da quelle che affiorano nel nostro ordinamento. Naturalmente ci sono state eccezione: ma, appunto, di eccezioni si è finora trattato. Quasi tutte incentrate sull'esperienza nord-americana $^{3}$, come risulta anche dai programmi relativi ai corsi di diritto processuale penale comparato attivati in alcune università ${ }^{4}$.

Ora le cose stanno cambiando anche grazie alla propensione degli studiosi più giovani a frequentare università e istituti di ricerca stranieri. Molti dei dottorati di ricerca attivi presso le nostre università impongono periodi più o meno lunghi di ricerca all'estero, nel corso del triennio. Il crescente rilievo che per il diritto interno hanno le normative euro-unitarie (molte delle quali riguardanti il diritto processuale penale), le sentenze della Corte EDU e della Corte di Giustizia UE accresce, pur in via indiretta come si dirà, l'interesse (particolarmente dei più giovani) per le esperienze giuridiche di altri ordinamenti.

Sta dunque formandosi una nuova leva di studiosi che - facile prevederlo - faranno della comparazione uno strumento imprescindibile sia per auspicabili spunti di riforma del sistema processuale, sia per una miglior

2 G. Spangher, Considerazioni sul processo penale italiano, Giappichelli, Torino, 2015, p. 1 e 2.

3 Per quanto concerne la comparazione con gli USA meritano di essere ricordati i nomi di Ennio Amodio, Rosanna Gambini, Vittorio Fanchiotti, Luca Marafioti, Vania Patanè. All'ordinamento processuale francese hanno prestato particolare attenzione Mario Chiavario, Gabriella Aimonetto, Novella Galantini.

4 Da una rapida consultazione dei rispettivi siti WEB risultano attivati corsi di questo nei seguenti atenei: Bologna, Catania, Foggia, Genova, Milano Statale, Padova, Palermo, Torino, Urbino; M. Miraglia, Garanzie costituzionali nel processo penale statunitense: tendenze e riflessioni, Giappichelli, 2008 
comprensione del diritto positivo interno, sia, infine, per adeguare le applicazioni della legge statale alle istanze euro-unitarie e alla giurisprudenza delle Corti europee.

Ogni cultore del diritto interno dovrebbe avere - io credo - una naturale tensione conoscitiva verso ordinamenti diversi: tanto più in un contesto come l'attuale, dove gli ordinamenti statali sono compenetrati di normative sovranazionali che pongono problemi pratici, ad esempio, sul terreno dell'armonizzazione dei sistemi penali e processuali, in vista di una più efficace cooperazione giudiziaria. Siccome però non sarebbe utile (né si può ragionevolmente pretendere) una comparazione ad amplissimo raggio tale da riferirsi a molteplici esperienze diverse da quella di partenza, occorrerà selezionare con consapevole avvedutezza l'ordinamento o gli ordinamenti cui riferirsi per un proficuo confronto.

È questo il primo nucleo problematico che il comparatista si trova davanti e su questo converrà fermare l'attenzione nel prossimo paragrafo.

Altri temi meritevoli di qualche chiosa sono le finalità e gli oggetti della possibile comparazione: perché comparare? E cosa, precisamente, entra nel fuoco della comparazione?

2) Realtà rilevanti.- Quanto sin qui detto si regge su un sottinteso che va svelato: la comparazione si fa con altri ordinamenti vigenti; nel caso del processo penale, con sistemi processuali e ordinamenti giudiziari di altre realtà statuali contemporanee. In realtà accanto a questa - definibile come comparazione sincronica - sappiamo che esiste una comparazione diacronica, orientata al confronto con sistemi pre-vigenti, più o meno lontani nel tempo. L'effetto di "distanza” tipico dell'approccio comparatistico, viene in altre parole ricercato sul piano del passato, vivificando mentalmente esperienze del passato $^{5}$. Bisogna anzi riconoscere che i migliori contributi comparatistici della dottrina processualpenalistica italiana provengono proprio da questo tipo di approccio ${ }^{6}$.

5 Che la comparazione si avvalga dello "punto di vista esterno" è affermazione sulla quale ferma opportunamente l'attenzione A. Somma, Introduzione al diritto comparato, Giappichelli, Torino, 2é ed., 2019, p. 21 ss.

6 Di grande rilievo, per l'influenza su autori più giovani, gli studi che Franco Cordero ha dedicato agli sfondi storici dei principali istituti processuali. Spunti di riflessione sono contenuti già nelle diverse edizioni del manuale di Procedura penale pubblicato da Giuffrè a partire dal 1966 e fino al 2011). Trattazioni più dettagliate — con frequenti riferimenti alla giustizia penale dell'Ancien Régime — sono contenute in altri scritti di carattere prettamente storico, fra i quali vale la pena citare Riti e sapienza del diritto, Bari-Roma, Laterza, 1985; La fabbrica della peste, Laterza, Bari-Roma, 1985, e Criminalia. Nascita dei sistemi penali, Laterza, Bari-Roma, 1986.

Accurate ricostruzioni storiche su taluni istituti della giustizia penale principalmente focalizzate sulla cultura illuministica affermatasi nella Francia rivoluzionaria si ritrovano in alcune pregevoli monografie pubblicate negli anni '70 e '80 del secolo scorso: Di particolare valore gli approfondimenti condotti da M. Nobili sull'attività decisoria del giudice penale (Il principio del libero convincimento del giudice, Milano, Giuffrè, 1974) e da P. Ferrua sul retroscena storico del principio del contraddittorio (Oralità del giudizio e letture di deposizioni testimoniali, Milano, Giuffré, 1981).

Volendo qui dar conto della sola produzione scientifica interna al settore disciplinare, si evita di citare studi prodotti dagli storici del diritto. Almeno un nome va tuttavia fatto per la grande eco che i 
La comparazione diacronica - come la sincronica - punta all'effetto di "straniamento", che costituisce la postura mentale tipica del comparatista. Guardare il proprio ordinamento dall'esterno (assumendo il punto di vista di realtà di altre epoche storiche o di altri contesti ordinamentali contemporanei) consente di guardare criticamente, dall'esterno, la propria realtà di provenienza, cogliendone tratti destinati a sfuggire all'osservazione di chi limita l'area dei propri interessi scientifici all'orizzonte del diritto positivo interno. C'è però il serio rischio che, nel calarsi in quell'altro contesto, si assumano postulati o punti di vista del tutto arbitrari o infondati, tali da rendere inutile, se non addirittura nocivo, lo sforzo comparatistico. Chi compara due o più entità deve, in altre parole, conoscere bene i termini del confronto e non farsi troppo influenzare da passioni politico-ideologiche o da finalità etiche, sempre presenti nella riflessione giuridica. Compito difficilissimo nella comparazione diacronica, perché le ricostruzioni storiche impongono di "spostarsi mentalmente" in una situazione politico-storico-sociale consegnata al passato, della quale sopravvivono tracce più o meno visibili. Occorre saper ben maneggiare le fonti storiche: cosa che non sempre gli studiosi di diritto positivo sanno fare con la dovuta abilità. Più si risale all'indietro nel tempo e più diventa arduo azzardare paragoni convincenti. Accade così che la ricostruzione di certi istituti al centro dello sforzo comparativo avvenga sul presupposto di assunti di partenza che il lettore è invitato a condividere come postulati della proposta comparazione. Un esempio chiarirà quanto intendo affermare. In una ricostruzione storica della regola BARD (Beyond Any Reasonable Doubt) James Q. Whitman muove dal seguente interrogativo: come si spiega che il superamento delle ordalie (nel lontano 1215) abbia portato, secoli dopo (fine Settecento), a formalizzare il principio dell'“al di là di ogni ragionevole dubbio" per la prima volta nell'Old Baily londinese ${ }^{7}$. La spiegazione andrebbe individuata - secondo l'autore - nell'esigenza - molto avvertita dai giurati dell'epoca, chiamati a giudicare delitti capitali - di essere liberati dal peso morale (moral anxiety) che avrebbe accompagnato l'eventuale condanna. L'ordalia permetteva di trasferire sulla divinità questo pesante fardello. Venuta meno la pratica ordalica per l'editto pontificio di Innocenzo III, quel peso è gravato interamente sulle spalle di uomini in carne e ossa. Per liberarli dal possibile senso di colpa conseguente alla possibile condanna, si è andata gradualmente affermando la ricordata regola di giudizio. La conclusione dello studio coincide col postulato di partenza. Quel postulato consente di mettere in fila le fonti da cui trarre una raffica di conferme, secondo un

\footnotetext{
suoi studi hanno avuto sui cultori del diritto e della procedura penale delle generazioni scientificamente attive negli anni '70-'90 del secolo scorso. Alludo a Mario Sbriccoli, che, a partire dalla monografia sulla giustizia politica (Crimen laesae maiestatis. Il problema del reato politico alle soglie della scienza penalistica moderna, Milano, Giuffrè, 1974) è stato a lungo un interlocutore autorevole e ascoltato degli studiosi di diritto positivo. Per aver un'idea della quantità e profondità di spunti offerti ai penalisti contemporanei da questo autore basta consultare gli scritti pubblicati nel volume (postumo) M. Sbriccoli, Storia del diritto penale e della giustizia. Scritti editi e inediti (1972-2007), Milano, Giuffrè, 2009.

${ }^{7}$ Cfr. J. Whitman, The Origins of Reasonable Doubt: Theological Roots of the Criminal Trial, Yale University Press, 2008.
} 
percorso argomentativo di grande interesse e fascino, ma sostanzialmente tautologico. Tutto crolla, sa se non si condivide quel punto di partenza, abilmente posto da Whitman a fondamento della sua ipotesi ricostruttiva ${ }^{8}$. Ma, quand'anche l'ipotesi di lavoro fosse smentita o risultasse poco convincente, l'opera di comparazione, se condotta con abilità nel maneggio delle fonti storiche, potrebbe comunque accendere delle luci utili alla comprensione di istituti e fenomeni dell'attualità contemporanea. Ad esempio, qualunque cosa si pensi del citato volume di Whitman, è innegabile il valore dello studio in esso condotto, quanto meno con riguardo all'originalità dell'idea di partenza e alla coerenza con la quale essa è stata coltivata e documentata nell'intera ricerca. Si pone così una tesi seriamente sostenuta che attende di essere vagliata criticamente e che contribuisce ad accrescere la conoscenza dell'oggetto studiato anche per chi non la dovesse condividere.

Del resto, è comprensibile che, per ovviare alla difficoltà (o impossibilità) di dislocarsi e, per così dire, immergersi in epoche lontane o lontanissime, della cui organizzazione politico-sociale, delle cui pratiche giuridiche e dottrine siamo in larga parte ignari, si elabora una tesi di lavoro, da verificare come conclusione della ricerca alla luce di tracce documentali poste a fondamento di ipotesi ricostruttive buone fino alla prossima smentita. La comparazione diacronica va condotta con grande prudenza, onde evitare travisamenti o analogie tanto suggestive quanto fuorvianti. Ma - quando ben riuscita essa rende servizi di straordinaria utilità alla comprensione di istituti di epoche passate, mettendone in evidenza possibili linee di continuità con istituti attuali. Può esser portato ad esempio, a tal riguardo, il primo volume della Cassazione civile di Piero Calamandrei: una magistrale ricostruzione storica (a partire dal diritto romano) del ricorso di legittimità (istituto giudiziario), che nel corso del tempo si salda con la Corte di cassazione (istituto politico). Benché risalente a un secolo fa, quel volume rappresenta ancor oggi una lettura imprescindibile per chi intenda approfondire la funzione e l'attività della Corte di cassazione in ambito sia civile, sia penale. Anche le opere migliori, naturalmente, invecchiano. Bisogna però ammettere - pensando al citato contributo comparatistico di Calamandrei - che chi oggi si occupa di funzione nomofilattica del giudice di legittimità, troverà utile la distinzione fra ius litigatoris e ius constitutionis; chi si interroga sulle origini degli errores in procedendo come motivi di annullamento della sentenza di merito, troverà illuminanti certe affinità con la (romana) querela nullitatis; chi intende vagliare criticamente il rapporto giudice/legge, avrà occasione di meditare sulle singolari ascendenze della Cour de cassation francese con figure dell'Ancien Régime quale il Conseil du roi e di riflettere sul tratto politico che ancora caratterizza questo organismo giudiziario anche nella sua versione italiana.

\footnotetext{
8 Questa la critica mossa da Th. P. Gallanis, Reasonable Doubt and the Origins of Criminal Trial, in The University of Chicago Law Review, 2009, 961 ss., che pur mostra di apprezzare lo sforzo ricostruttivo intrapreso da J. Q. Whitman.
} 
La comparazione sincronica presenta minori rischi di travisamento, purché lo studioso sappia immergersi nella realtà prescelta come termine di raffronto. Evidentemente è impossibile allargare lo sguardo a una molteplicità di ordinamenti. Una comparazione ad amplissimo raggio darebbe peraltro risultati di scarsa utilità sia teorica (vale a dire in termini di comprensione degli istituti presi di mira), sia pratica (vale a dire, in termini di proposte di riforma del diritto interno). Andrà fatta una scelta, prediligendo quelle realtà che offrono un ricco materiale di confronto.

Ciò pone il problema del "perché" valga la pena comparare e del "cosa" meriti di essere comparato.

3) Finalità della comparazione (in ambito processuale penale).- Volgendo lo sguardo alla letteratura italiana, si nota che la comparazione in ambito processuale penale ha puntato a diverse finalità.

La comparazione di tipo diacronico punta alla miglior comprensione dell'attualità. Ha quindi finalità principalmente teorico-dottrinali, occasionalmente idonee a fornire spunti critici sugli assetti normativi vigenti o su orientamenti giurisprudenziali da approvare/censurare. Serve ad acquisire consapevolezza dell'origine remota di certi istituti, allo scopo di favorire l'uso di categorie concettuali risalenti, che, nel tempo e per il mutare delle condizioni socio-politiche, possono aver subito rilevanti variazioni di significato ${ }^{9}$. Questo tipo di comparazione si presta sovente ad essere impiegata o strumentalizzata per ragioni politico-culturali, magari motivate dall'esigenza (avvertita come impegno civile) di prendere le distanze o di ripudiare pratiche processuali che, pur appartenenti ad epoche passate, si percepiscono come incombenti sull'epoca attuale ${ }^{10}$.

La comparazione di tipo sincronico viene intrapresa per finalità analoghe, ma, come già accennato, rispetto all'altra ha il vantaggio di riferirsi a ordinamenti vigenti, suscettibili di essere osservati direttamente dallo studioso. Finalità teorico-dottrinali (imparare qualcosa dagli studi di autori stranieri che si occupano di istituti e temi rilevanti per lo studioso di diritto positivo

\footnotetext{
9 Ad esempio, la monografia di M. Nobili su Il principio del libero convincimento del giudice (già citato supra, nota 6), offre una panoramica sul passaggio dalle pratiche criminali inquisitorie basate sulla prova legale alle normative processuali aperte all'intimo convincimento fra XVIII e XIX secolo; e denuncia, al contempo, la possibile degenerazione (in arbitrio) che una simile apertura ha di fatto comportato. P. Ferrua, con la sua monografia su Oralità del giudizio e letture di deposizioni testimoniali (già citato supra nota 6) ricostruisce il provvisorio affermarsi del principio di oralità nel cruciale ventennio che separa l'esperienza della rivoluzione francese dalla redazione del code d'instruction criminelle, allo scopo di evidenziare quanto sia difficoltoso affermare un modello adversary di processo penale in un contesto giuridico permeato di cultura inquisitoria.

Pubblicati in un periodo di forti contrasti ideologici e di particolare fervore degli studi processuali penali, entrambi questi scritti hanno avuto notevole influenza sulle discussioni intorno alla riforma del codice di procedura penale.

10 Fra gli autori italiani vanno ricordati, a questo riguardo, i ponderosi ed eruditissimi studi di F. Cordero (già citati supra nota 6), pubblicati verso la metà degli anni ' 80 , quando sembrava tramontata la riforma processuale che sarebbe maturata nel 1988. Si può cogliere in quegli studi l'intenzione di ammonire la dottrina processualistica circa i rischi di involuzioni inquisitorie che avrebbero caratterizzato un sistema di giustizia penale del quale appariva ardua la riforma in senso adversary.
} 
interno) e finalità lato sensu politiche, animate dall'intento di suggerire proposte di riforma.

Si pensi, per stare all'attualità, ai molti scritti che circolano in questi giorni sulla prescrizione del reato, su come questa delicata materia è regolata negli USA, in Francia, in Germania, in Spagna etc. Vi si illustrano soluzioni diverse dalla nostra (oggetto di vivaci controversie); soluzioni dalle quali ci si aspettano spunti di riflessione volti a sostenere o a smentire la tesi della "prescrizione sine die" dopo la sentenza di primo grado. Si tratta, spesso, di prese di posizione superficiali, prive di quel retroterra di studio e approfondimento dell'ordinamento "straniero" che dovrebbe caratterizzare ogni seria indagine comparata. Ciò nondimeno, il ricorso (pur superficiale) all'argomento comparativo circa il tema appena esemplificato segnala la naturale curiosità e il comprensibile desiderio di sapere "cosa fanno gli altri", quando ci si trova di fronte a questioni o problemi che si ritiene siano comuni agli ordinamenti contemporanei.

Non ci si può improvvisare nella comparazione. Il discorso vale in particolare per quegli studiosi di diritto positivo interno, che avvertono con particolare urgenza la curiosità e il desiderio di confrontarsi con altre esperienze. Chi si impegna in questo tipo di approccio scientifico deve conoscere bene non solo l'ordinamento politico costituzionale del Paese preso a termine di paragone, ma anche la lingua, la storia, i costumi, le espressioni culturali e letterarie dell'altra realtà. Anche per questa ragione, una vera comparazione (sincronica) non la si può fare ad ampio raggio.

Conviene scegliere con oculatezza l'ordinamento da "studiare" e lavorare su quello, in profondità, acquisendo informazioni di dettaglio simili a quelle che lo studioso possiede del proprio ordinamento. Non solo. È opportuno che la scelta cada su un ordinamento non troppo distante dal nostro sia per tradizione giuridico-culturale ${ }^{11}$, sia per dimensioni abitative e territoriali ${ }^{12}$. $\mathrm{E}$ andranno preferite quelle esperienze che - oltre a un'articolata realtà normativa - offrono una ricca riflessione dottrinale, capace di agevolare il comparatista nella messa a fuoco delle norme anche nel loro momento applicativo $^{13}$.

11 Studiare i sistemi penali del mondo islamico può certo fornire informazioni preziose per il comparatista, ma con elevatissimi rischi di fraintendimento e, perciò, di manipolazione del dato acquisito.

12 Anche la comparazione con realtà minuscole (quali la Repubblica di S. Marino, il principato di Andorra, il principato del Liechtenstein), certamente dotati di un proprio sistema di giustizia penale, presenta rischi di fraintendimento e manipolazione certo diversi da quelli segnalati alla nota precedente, ma tali da sconsigliare, in linea generale, azzardati paragoni.

13 Questa è verosimilmente la ragione per la quale i cultori del diritto e del processo penale hanno volto la loro attenzione preferibilmente al mondo tedesco, notoriamente caratterizzato da una fluviale produzione dottrinale. Di minor interesse appare l'esperienza francese per (si può dire) l'assenza di una riflessione dottrinale sugli istituti e per lo stile stringato (poco argomentato) delle decisioni giurisprudenziali. La causa di questo sta forse in quell'art. 5 del Code Napoleon, tuttora in vigore nel code civil francese, che vieta al giudice di decidere i casi a lui sottoposti facendo leva su massime generalizzanti (Il est défendu aux juges de prononcer par voie de disposition générale et réglementaire sur les causes qui leur sont soumises.). Affiora la diffidenza del legislatore di fronte alla interpretazione giudiziale. Questo 
Come accennato in apertura, non sono numerosi, in Italia, gli autori che hanno coltivato passioni e curiosità per gli ordinamenti stranieri. Limitandoci alla produzione bibliografica degli ultimi decenni, quasi tutti hanno volto la loro attenzione ai sistemi di common law, anche in ragione della svolta in senso adversary impressa alla riforma processuale italiana negli anni Ottanta del secolo scorso ${ }^{14}$.

Oltre alla segnalata finalità pratica (suggerire soluzioni normative), la comparazione sincronica può avere anche finalità teorico-dottrinali: acquisire o prendere a prestito categorie concettuali maturate nell'ambito di altre esperienze giuridiche. Al riguardo si possono fornire due esempi assai significativi per il settore disciplinare della "procedura penale". Alludo a due monografie, entrambe pubblicate verso la metà degli anni '50 del secolo scorso, che hanno costituito - si può dire - il punto di partenza metodologico per la generazione di studiosi del diritto processuale penale che per prima sperimentò concretamente l'autonomia didattica della disciplina ${ }^{15}$. Si tratta del volume di Giovanni Conso, intitolato I fatti giuridici processuali penali (Milano, Giuffrè, 1955) e del volume di Franco Cordero, Le situazioni soggettive del processo penale, Torino, Giappichelli, 1956).

Basta sfogliare lo studio di Conso, con attenzione all'apparato bibliografico e di note, per rendersi conto di quanto l'autore sia debitore a studiosi italiani di altre discipline ${ }^{16}$ o a studiosi di lingua tedesca di concetti-chiave

principio ha un suo corollario nella regola che vieta al giudice di citare nelle sentenze opinioni dottrinali (dalle quali potrebbero essere tratte quelle massime generalizzanti). Un divieto che vale anche da noi (art. 118 comma 3 disp. att. c.p.c.), ma che il giudice italiano interpreta restrittivamente, intendendo quel divieto come riferito ai soli nomi degli autori, non alle loro opinioni. Sicché abbondano nelle motivazioni delle sentenze italiane (civile e penali) affermazioni del tipo "è stato affermato in dottrina" o "sostiene autorevole dottrina". Un simile divieto, come si sa, non vale per i giudici tedeschi, i quali, anzi, col citare autori e opere a sostegno delle interpretazioni prescelte, ritengono di impreziosire le loro sentenze. Le citazioni dottrinali nei provvedimenti giudiziari alimentano, a ben vedere, il dialogo fra dottrina e giurisprudenza, col risultato — se la dottrina è di buona qualità — di arricchire entrambe. L'inserimento di opinioni dottrinali nelle motivazioni dei giudici ha altresì l'effetto di estendere l'area dei possibili "oggetti" della comparazione, come risulterà chiaro da quanto si dirà subito di seguito.

${ }_{14}$ Vale la pena citare in particolare M. Scaparone, Common law e processo penale, Milano, Giuffrè, 1971; E. Amodio (a cura di) I giudici senza toga: esperienze e prospettive della partecipazione popolare ai giudizi penali, Milano, Giuffrè, 1979; La cross-examination: strategie e tecniche, Milano, Giuffrè, 1990; E. Amodio-M. Ch. Bassiouni, Il processo penale negli Stati Uniti d'America, Giuffrè, Milano, 1988. Una ampia rassegna dei contributi comparatistici in ambito processuale penale nell'arco di un secolo abbondante di storia italiana è offerta da E. Amodio, La procedura penale comparata tra istanze di riforma e chiusure ideologiche (1870-1989), in Riv. it. dir. e proc. pen. 1999, p. 1338 ss.

15 L'insegnamento della procedura penale era stato elevato al rango di disciplina autonoma a livello universitario nel 1938. Tuttavia, fino agli inizi degli anni Sessanta, l'insegnamento fu quasi sempre assegnato, per affidamento, ai professori di diritto penale. Rare le eccezioni: Grispigni, Carnelutti e poi Leone a Roma, Manzini a Torino. I primi concorsi dedicati esclusivamente al settore disciplinare oggi identificato con la sigla IUS/16 furono banditi nella seconda metà degli '50. In quel torno di tempo escono le due monografie menzionate nel testo, che, per alcuni decenni, faranno da guida ai nuovi studiosi della disciplina.

16 È comprensibile che, nel momento in cui una disciplina cerca di rifondarsi, chi si cimenta con un simile compito avverta l'esigenza di prendere a prestito concetti già sperimentati in ambiti disciplinari contigui come il diritto processuale civile e il diritto amministrativo, entrambi per verità largamente influenzati dalla dogmatica tedesca a partire dalla fine del XIX secolo (per il diritto ammi- 
quali "presupposti processuali", "rapporto giuridico-processuale", "fattispecie a complesse a formazione successiva". Una visione del processo penale saldamente ancorata ai principi del tecnicismo giuridico, già affermatosi da decenni in altre discipline giuridiche ${ }^{17}$.

Analoghi rilievi possono essere fatti per il citato volume di Cordero. C'è, in esso, l'ambiziosa pretesa di costruire una dogmatica processuale alternativa a quella proposta da Conso, ispirandosi al pensiero di James Goldschmidt e, in particolare, alla monumentale monografia Der Prozess als Rechtslage: eine Kritik des prozessualen Denkens 18. Il grande processualista tedesco, seriamente preoccupato per la crisi di legalità che aveva investito la repubblica weimariana, era andato elaborando una teoria processuale innovativa che, superando le astrattezze del tecnicismo giuridico, metteva in primo piano le "situazioni giuridiche" che potevano succedersi nell'iter procedurale, vale a dire, i concreti rapporti di forza e di potere fra i soggetti processuali, nel prisma delle regole che disciplinano il giudizio (sia civile, sia penale): una prospettiva di grande interesse per il realismo che la caratterizzava e sul quale si poteva far leva per una serrata critica politica di istituti processuali criticati come lascito di una cultura autoritaria e antidemocratica. Nella "situazione giuridica” confluiscono le norme che governano l'iter processuale e il miscuglio di aspirazioni, speranze, timori, possibilità che quelle stesse norme contribuiscono quotidianamente ad alimentare.

Le menzionate categorie concettuali apprese per via di comparazione sono ormai uscite dal lessico del processual-penalista. Esse hanno tuttavia avuto una importanza notevolissima nella nascita e nello sviluppo di una moderna cultura processual-penalistica in Italia e, in definitiva, nella formazione dei docenti di procedura penale ${ }^{19}$.

4) Gli oggetti della comparazione. - Una discutibile opinione limita l'oggetto della comparazione agli assetti normativi di diversi ordinamenti ${ }^{20}$. Serve davvero a poco confrontare fra loro le regole che governano certi istituti o certi fenomeni in ordinamenti diversi ${ }^{21}$. Da quanto detto al paragrafo prece-

nistrativo) e dall'inizio del xx secolo (per il diritto processuale civile). In ogni caso, anche il trarre ispirazione da categorie concettuali maturate in altre discipline di diritto interno è — a ben vedere — una forma di comparazione.

17 Per ulteriori informazioni sull'affermarsi del tecnicismo giuridico nelle discipline giuridiche e, in particolare, in quelle penalistiche, si rinvia a R. Orlandi, La prolusione di Rocco e le dottrine del processo penale, in Criminalia 2010, 2011, p. 207 ss.

18 Berlin, Springer, 1925.

19 Giovanni Conso esercitò una grande influenza sugli studi processuali penali, anche grazie alla collana di studi da lui diretta per l'editore Giuffrè (a partire dal 1959). Quanto all'influenza di Cordero (che, tra l'altro, sarà il primo a pubblicare nella citata collana diretta da Conso), basterà citare il suo fortunato manuale Procedura penale (molteplici edizioni a partire da quella del 1966 fino all'ultima datata 2011), sul quale si sono formate più generazioni di processualisti.

$20 \mathrm{E}$, ad esempio, l'opinione palesata dall'autore citato supra, nota 2.

${ }^{21}$ Questo modo di fare comparazione era tipico della letteratura giuridica risalente a un secolo fa. Si prenda qualche voce enciclopedica del Digesto italiano. Ad esempio, nella voce Polizia giudiziaria (Dig. it., vol. XVIII, parte II, Torino, UTET, 1924, p. 1057 ss.) l'autore (Francesco Carfora) dà sommariamente conto di ben quindici ordinamenti stranieri (nell'ordine, Francia, Belgio, Austria, Ungheria, 
dente dovrebbe essere chiaro che l'oggetto della comparazione, oltre alle leggi scritte, sono anche le opinioni che su quelle leggi si formano in giurisprudenza e in dottrina. Del resto, è assunto ormai pacificamente condiviso che le leggi valgono per come sono interpretate: vale a dire, per le opinioni che su di esse formula la giurisprudenza (in particolare, di legittimità), sovente sorretta su riflessioni e chiarimenti dottrinali.

Consapevole di questo, il comparatista moderno non può limitarsi a comparare assetti normativi o istituti regolati da norme legali o statutarie. Deve allungare lo sguardo a quelle prese di posizione e a quei concetti di origine giurisprudenziale e dottrinale che - con le dovute cautele - possono essere valorizzate per proposte di riforma, sia per affrontare questioni teoriche (dottrinali) o problemi applicativi (giurisprudenziali) di diritto interno. Qualche esempio servirà ad illustrare meglio quel che intendo.

Capita con una certa frequenza di veder evocato sia in dottrina sia in giurisprudenza il "principio di proporzionalità" con riferimento all'applicazione di norme processuali penali. Si tratta di un principio non scritto, utile per misurare il grado di comprimibilità di un diritto individuale, per motivi di prevenzione o repressione dei reati. Criterio di razionalità pratica, coerente con l'idea (familiare a uno Stato di diritto) che i "danni" e le "sofferenze" provocati dai pubblici poteri (amministrativi o giudiziari) vanno commisurati alla gravità della situazione di volta in volta affrontata; devono essere idonei a perseguire un fine legalmente stabilito e non devono eccedere la misura dello stretto necessario. Chi ha qualche frequentazione comparativa con gli ordinamenti di area tedesca sa (o dovrebbe sapere) che questo principio è il frutto di una lunga, raffinata elaborazione giurisprudenziale e dottrinale. In particolare, la Corte costituzionale tedesca, già a partire dai primi anni Cinquanta del secolo scorso, ne ha fatto un caposaldo del delicato bilanciamento fra tutela dei diritti individuali ed esigenze preventivo/repressive. La dottrina, dal canto suo, ha contribuito ad affinarlo ulteriormente, facendone spesso argomento di critica verso soluzioni normative di tipo emergenziale, ad esempio in tema di interventi contrassegnati da particolare rigore nel perseguimento e nella prevenzione di delitti gravissimi (criminalità organizzata, terrorismo). Il principio viene spesso richiamato (senza essere definito e quindi, dandone per scontato il contenuto) in molte fonti normative euro-unitarie ${ }^{22}$ oltre che nella giurisprudenza delle due corti europee ${ }^{23}$. Anche la

\footnotetext{
Germania, Canton Ticino, Spagna, Norvegia, Inghilterra, India, Stato di New York, Uruguay, Venezuela, Bolivia, Sudan). Si sostiene che la selezione è stata fatta individuando "i codici più importanti come esempi dei diversi sistemi”. Ci si rende poi conto che questa molteplicità di informazioni rischia di disorientare il povero lettore e si arriva quindi alla conclusione che, in realtà, conviene ridurre il confronto a tre tipi principali: il francese, il tedesco, l'inglese (ivi, p. 1064, par. 42). Qui — dove in realtà dovrebbe iniziare - l'analisi comparata si interrompe. È un esempio, se si vuole estremo, di come la comparazione non vada fatta rivelandosi pressoché inutile.

${ }_{22}$ Ad es., nella direttiva 2014/42/UE, del 3 aprile 2014, relativa al congelamento e alla confisca dei beni strumentali e dei proventi da reato nell'Unione Europea.

${ }^{23}$ Per stare ad alcuni casi recenti: Corte EDU, $2^{\circ}$ sez., 10 dicembre 2019, Kavala v. Turkey in tema di misure limitative di libertà personale (art. 5 CEDU); Corte EDU, $5^{\circ}$ sez, 30 gennaio 2020, Breyer v.
} 
nostra Corte costituzionale ne fa un uso crescente in materia processuale penale ${ }^{24}$. Orbene, lo studioso che conosce la paziente elaborazione che del proportionality standard hanno fatto i giuristi tedeschi ha un indubbio vantaggio su chi ignora tutto quel dibattito e le sottili distinzioni cui esso è approdato. Non è detto che si debba dar ragione a chi maneggia da decenni un simile principio: si ammetterà però che chi conosce l'origine di un criterio interpretativo dottrinale o giurisprudenziale, avendo un'idea piuttosto precisa delle molte occasioni in cui lo si è impiegato, ha maggiori possibilità di tradurlo in (o di suggerirne la) pratica applicazione nel proprio diritto interno ${ }^{25}$.

Dall'esperienza tedesca ci viene un altro esempio di utile comparazione avente ad oggetto una creazione giurisprudenziale. Alludo alla pratica dei captatori informatici di cui si è discusso anche in Italia, soprattutto dopo che la nostra Corte di cassazione - in mancanza di una normativa legale ad hoc - ne ha ammesso l'uso, pur a certe condizioni ${ }^{26}$. Questa sentenza della Cassazione risale al 2016, benché sia noto da tempo che questi micidiali strumenti investigativi sono di fatto nella disponibilità delle forze di polizia. Per rendersene conto, era sufficiente spingere lo sguardo al di là delle Alpi, dove la Corte costituzionale tedesca - già nel 2008 - aveva affrontato il problema, se e in che misura fosse legittimo l'uso di malware informatico come mezzo non solo per intercettare comunicazioni interpersonali, ma anche per ispezionare le memorie dei dispositivi elettronici che siamo soliti maneggiare quotidianamente (smartphone, tablet, personal computer). L'attenzione al dato comparativo avrebbe consentito di evitare un duplice inconveniente: il notevole ritardo dell'intervento legislativo ${ }^{27}$ e la sua limitazione all'uso dei captatori informatici come mezzi per intercettare comunicazioni, non anche come strumenti per controllare a distanza la messe di documenti e dati personali archiviati nei nostri hard disk 28.

5) Utilità attuale della comparazione, nuovi compiti e conclusioni. - Fino a qualche tempo fa si poteva sostenere che la comparazione (soprattutto quella sincronica) rappresentava un di più rispetto ai tradizionali compiti dello stu-

Germany, in tema di misure limitative del diritto alla riservatezza (art. 8 CEDU); CGUE, Grande Sezione, sentenza 2 ottobre 2018, causa C-207/16, anch'essa in tema di misure limitative del diritto alla riservatezza (artt. 7 e 8 Carta dei dir. Fondamentali UE).

${ }^{24}$ Ad esempio, nelle sentenze che hanno dichiarato parzialmente illegittimo l'art. 275 comma 3, nella parte in cui stabiliva un incondizionato automatismo nella scelta della custodia cautelare in carcere per alcuni tipi di delitti che avrebbero potuto ragionevolmente comportare una misura meno afflittiva (sent. nr. 265 del 2010; sent. nr. 231 del 2011; sent. nr. 110 del 2012; sent. nr. 57 e 213 del 2013; sent. nr. 48 del 2015).

${ }^{25}$ Una illustrazione del principio di proporzionalità che tiene conto del ricco dibattito tedesco è offerta da D. Negri, Compressione dei diritti di libertà e principio di proporzionalità davanti alle sfide del processo penale contemporaneo, in Riv. it. dir. e proc. pen. 2020, p. 3 ss.

26 Cass. Sez. unite $1^{\circ}$ luglio 2016 (c.c. 28 aprile 2016), Scurato, in CED, RV 266905.

27 Con il d. lgs. n. 216 del 2017, ripetutamente prorogato fino al $1^{\circ}$ maggio 2020 e modificato dal d.l. n. 161 del 2019 convertito nella l. n. 7 del 28 febbraio 2020.

28 Per una critica delle insufficienze della normativa italiana sui captatori informatici sia consentito rinviare a R. Orlandi, Usi investigativi dei captatori informatici. Criticità e inadeguatezza di una recente riforma, in Riv. it. dir. e proc. pen. 2018, p. 538 ss. 
dioso di diritto positivo interno; una sorta di optional che - se ben coltivato poteva rendere più convincenti le sue opinioni, accrescendone il pregio. Conoscere il diritto straniero poteva cioè servire a capire meglio il proprio, così come imparare un'altra lingua predispone a usare con maggior consapevolezza il proprio idioma principale.

Questa idea, che poteva avere un suo fondamento quando si predicava l'esistenza di un "diritto obiettivo nazionale" ${ }^{29}$, appare superata a misura che l'ordinamento statale accetta di entrare in una relazione vitale e dialettica con ordinamenti sovranazionali che ne limitano la sovranità. La correlativa soggezione alle direttive euro-unitarie e alla giurisdizione delle alte Corti europee espone l'ordinamento italiano a quel graduale processo di armonizzazione normativa, già da tempo in atto anche nel settore del diritto e della procedura penale. Ciò implica l'interessata conoscenza di altri ordinamenti per ragioni non solo teorico-dottrinali, ma altresì pratico-applicative. Qualche esempio.

In un caso riguardante l'esecuzione di mandato d'arresto europeo, la Corte di cassazione si trovò di fronte al seguente quesito: se l'assenza di termini di durata della custodia cautelare nell'ordinamento dello Stato richiedente (la Germania) fosse di ostacolo alla consegna della persona arrestata in Italia. La normativa italiana che attua la decisione-quadro sul mandato d'arresto europeo prevede, in effetti, il rifiuto di consegna, quando la normativa processuale dello Stato richiedente non prevede termini massimi di durata della misura limitativa di libertà ${ }^{30}$. La Corte di cassazione ha dunque dovuto effettuare una ricognizione della normativa processuale tedesca, per stabilire se vi fosse o meno contrasto con la normativa italiana. La Strafprozessordnung non prevede (come il c.p.p. italiano) termini rigidi di durata delle misure di coercizione personale; impone tuttavia al giudice di verificare, anche d'ufficio, almeno ogni sei mesi, se sussistono le condizioni per mantenerle in esecuzione. In altre parole, un termine di durata non è previsto, ma un controllo sui tempi della custodia cautelare è assicurato e tanto è bastato a orientare le sezioni unite della nostra Cassazione ${ }^{31}$ a esprimersi in favore della consegna ${ }^{32}$. Un bel caso di uso della comparazione a fini direttamente applicativi.

29 Espressione che ancora figura nell'art. 65 del nostro ord. giudiziario.

30 Art. 18 lett. e) l. nr. 69 del 2005.

31 Sent. del 5 febbraio 2007, Ramoci, in CED, RV 235348.

32 Questo il principio di diritto affermato in quella sentenza: «In materia di mandato di arresto europeo, con riguardo alla previsione dell'art. 18 lett. e) della legge 22 aprile 2005, n. 69, [...] l'autorità giudiziaria italiana deve verificare, ai fini della consegna, se nella legislazione dello Stato membro di emissione sia espressamente fissato un termine di durata della custodia cautelare fino alla sentenza di condanna di primo grado, o, in mancanza, se un limite temporale implicito sia comunque desumibile da altri meccanismi processuali che instaurino, obbligatoriamente e con cadenze predeterminate, un controllo giurisdizionale funzionale alla legittima prosecuzione della custodia cautelare o, in alternativa, alla estinzione della stessa». Nella parte precedente della motivazione la Corte si era premurata di dare conto - con tecnica comparativa — dello stato della normativa tedesca sui termini di durata della custodia carceraria. 
Altri esempi atti a illustrare l'uso della comparazione a fini interpretativi vengono dalla tendenza crescente a recepire i contenuti di decisioni emanate dalla Corte EDU, quando queste riguardano ordinamenti diversi da quello italiano. Che la Corte di Strasburgo condanni il Regno Unito, la Romania o la Turchia potrebbe apparire ininfluente per i giudici italiani. Ma vi sono situazioni in cui — nonostante la diversità degli ordinamenti penali e processuali — quel che Strasburgo dice a "nuora" dev'essere inteso anche da "suocera" 33. $\grave{E}$ accaduto in tema di diritto dell'imputato prosciolto in primo grado a non essere condannato in appello, se non dopo una ri-assunzione delle prove a suo carico. La circostanza che la Corte EDU si fosse espressa in favore di tale diritto (desumibile dall'art $6 \mathrm{CEDU}$ ), ha indotto a un certo punto anche la nostra Corte di cassazione ad allinearsi a quell'orientamento ${ }^{34}$, benché la legge processuale non contenesse alcun cenno al riguardo ${ }^{35}$.

La situazione politico-costituzionale nella quale siamo oggi immersi ci porta quasi forzatamente a conoscere dettagli di ordinamenti stranieri che non lasciano indifferenti né gli studiosi, né i pratici del diritto. Ogni volta che gli studiosi, i magistrati, gli avvocati leggono una sentenza della Corte EDU o della Corte di giustizia UE apprendiamo qualcosa degli ordinamenti con i quali abbiamo relazioni mediate da fonti sovranazionali. È cambiato lo scenario. La comparazione (sincronica) è divenuta elemento essenziale nella formazione del giurista positivo contemporaneo. Sarà ancora prezioso (soprattutto sul piano metodologico) il contributo dei "comparatisti tout court". Spetta però ai cultori degli altri settori disciplinari di diritto positivo aprirsi alla comparazione, quanto meno nell'intento di osservare e sorvegliare il processo di armonizzazione che da tempo sta interessando i Paesi europei sotto il segno della Convenzione europea dei diritti umani e del Trattato di Lisbona.

33 L'attenzione ai principi della CEDU e alla corrispondente giurisprudenza della Corte EDU esige un approccio comparatistico, in quanto implica sovente l'attitudine a spostare il proprio punto di vista, assumendo quello dello Stato (straniero) chiamato in giudizio. Si può dire che la giurisprudenza della Corte EDU è ottima palestra di comparazione. Infatti, i processualisti che si sono cimentati con i principi della Convenzione europea dei diritti umani e con la correlativa declinazione giurisprudenziale sono stati "naturalmente" caratterizzati dallo straniamento tipico della cultura comparatistica: valga per tutti l'esempio di M. Chiavario che, già con la sua prima monografia (La convenzione europea dei diritti dell'uomo nel sistema delle fonti normative in materie penale, Giuffrè, Milano, 1969) può essere considerato un pioniere della comparazione processuale penale in ambito italiano.

34 Cass. sez. unite, 6 luglio 2016, Dasgupta in CED, Rv. 267488.

In motivazione si citano alcuni precedenti, tutti relativi a ordinamenti stranieri: Corte EDU $3^{\circ}$ sezione, 8 luglio 2013, Manolachi c. Roumanie; Corte EDU, $3^{\circ}$ sezione, 5 novembre 2011, Dan v. Moldova; Corte EDU, $1^{\circ}$ sezione, 27 giugno 2000, Costantinescu v. Romania. Essendosi limitata a citare questi precedenti, non è dato capire in che misura la Corte di cassazione abbia "ragionato" comparativamente, nel tentativo di comprendere se le condanne inflitte a Moldavia e Romania esponessero anche la normativa italiana a una possibile censura sulla rinnovazione del dibattimento in appello. Tuttavia, considerata la conclusione qui essa perviene nell'affermare il principio di diritto, dobbiamo suppore che quel ragionamento sia stato fatto, restando poi inespresso nel testo della sentenza.

${ }_{35}$ La normativa processuale italiana è stata successivamente adeguata ai dicta della Corte di Strasburgo, recepiti dalle sezioni unite nella sentenza citata alla nota precedente: cfr. art. 603 comma 3-bis c.p.p., novellato da art. 1 comma 58 l. nr. 103 del 2017. 
C'è, in conclusione, una duplice ragione che spinge a coltivare lo spirito di comparazione.

La prima ragione è quella, per così dire, tradizionale. Si radica nella passione intellettuale e nella nostra personale curiosità su "come vanno le cose" in altri ordinamenti con i quali riteniamo proficuo confrontarci, per metterci in quella posizione di straniamento capace di farci meglio comprendere le regole e gli istituti del nostro ordinamento: questo vale per la comparazione sia diacronica, sia sincronica e resta un optional per il giurista di diritto positivo.

La seconda ragione sta nell'esigenza di osservare e sbrogliare gli intrecci che caratterizzano i rapporti fra sovranità statali nel contesto della "grande" e della "piccola" Europa: questo vale per la sola comparazione sincronica e dovrebbe rientrare nel bagaglio teorico e metodologico di ogni aggiornato cultore di Procedura penale. 